\title{
Extraction
}

\section{des canines incluses}

\section{RÉSUMÉ}

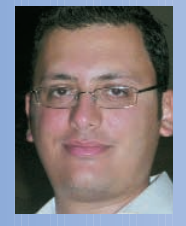

\section{Marc BARANES}

Interne en odontologie, Hôpital Charles Foix, Ivry-sur-Seine.

\section{Marie LAVAUD}

Assistante hospitalo-universitaire, Hôpital Charles Foix,

Ivry-sur-Seine.

\section{Louis MAMAN}

Chef du service d'odontologie, Hôpital Charles Foix,

Ivry-sur-Seine.

L'inclusion des canines est un phénomène fréquemment rencontré. Sa prise en charge est pluridisciplinaire. Elle requiert des compétences en chirurgie et en orthodontie. Lorsque l'extraction est indiquée, une analyse clinique et radiographique doit permettre de déterminer précisément la position de cette canine ainsi que ses rapports avec les dents et structures avoisinantes. Le choix du type d'anesthésie et de la technique chirurgicale se fera en fonction de I'analyse de ces paramètres.

canine incluse

- ectopie dentaire

- symphyse mentonnière
AOS 2008:244:377-393

DOI:10.1051/aos:2008046

C AEOS / EDP Sciences 


\section{Introduction}

Le diagnostic d'inclusion se fait par une évaluation clinique visant à rechercher des signes précis, comme la persistance sur l'arcade de la canine temporaire au-delà de 14 ans, l'absence d'espace d'éruption nécessaire entre la première prémolaire et I'incisive latérale, l'agénésie ou la microdontie de l'incisive latérale. L'examen clinique apprécie la mobilité de la canine temporaire et la présence d'une voussure incompressible vestibulaire, palatine ou linguale est recherchée. Les examens radiologiques (clichés rétroalvéolaires et occlusaux, radiographie panoramique et examen tomodensitométrique) permettront de visualiser précisément la hauteur et la profondeur d'inclusion de la canine, ses rapports de voisinage et la pré- sence d'éventuelles lésions associées: kyste péricoronaire, tumeur, résorptions diverses.

L'extraction d'une canine incluse est indiquée dans les cas d'impossibilité ou de refus du traitement conservateur orthodontique, lors des complications infectieuses, pseudotumorales ou lorsque l'inclusion est susceptible d'entrainer des risques pour les structures avoisinantes : résorption radiculaire, sinusite...

Elle doit s'accompagner d'une prise en charge globale esthétique et fonctionnelle, chacun connaissant l'implication majeure de la canine sur le plan occlusal.

Nous vous présentons trois cas cliniques pour lesquels la décision d'extraction d'une canine incluse a été prise.

\section{Extraction des canines incluses mandibulaires}

L'inclusion des canines mandibulaires est rare, elle est environ vingt fois moins fréquente que l'inclusion des canines maxillaires [1]. L'incidence de cette inclusion est évaluée à $0,4 \%$ de la population [1]. La migration intra-osseuse de la canine au cours de son évolution se fait généralement avec une inclinaison mésiale de la couronne puis la canine se redresse au contact de l'incisive latérale. Les deux tiers radiculaires sont édifiés au moment de l'émergence et le grand axe de la dent est vertical lorsque la couronne rejoint le plan d'occlusion [2]. En cas d'encombrement antérieur mandibulaire, la canine en évolution peut être retenue et le germe peut s'orienter de façon anormale: rotation axiale, inclinaison distale ou mésiale. Lorsque cette inclinaison mésiale fait un angle de $30^{\circ}$ avec le plan frontal passant par la canine, la correction orthodontique peut être envisagée. Si l'inclinaison est supérieure à $45^{\circ}$, le traitement orthodontique est contre-indiqué car il peut entrainer des séquelles sur les dents voisines [2]. Lorsque l'axe du germe fait un angle de 50 à $90^{\circ}$, la dent migre vers la région symphysaire ; ce phénomène est appelé transmigration. Celle-ci peut l'amener à traverser la ligne médiane. Plusieurs conditions sont favo- 
rables à la transmigration: I'inclinaison du germe supérieure à $30^{\circ}$, version coronovestibulaire des incisives mandibulaires et volume osseux symphysaire suffisant pour permettre la migration entre les racines des incisives et la corticale vestibulaire. La position de la canine incluse mandibulaire a été classifiée par Mupparapu en 2002 [3] (tableau I). L'extraction de la canine incluse mandibulaire en transmigration est indiquée en cas de complication infectieuse, manifestations pseudotumorales, manifestations algiques et risque mécanique pour le corps mandibulaire et les dents adjacentes.

\section{Situation clinique 1}

\section{> Présentation}

L'examen clinique d'un enfant de 15 ans, en bonne santé, révèle la persistance de 73 sur l'arcade alors que 43 a terminé son éruption (fig. 1). La radiographie panoramique (fig. 2) montre 33 en transmigration de classe 2 selon Mupparapu (tableau I) et I'inclusion de 13 et
23. Une hypertrophie du sac péricoronaire de 33 est observée. La téléradiographie de profil (fig. 3) met en évidence la position vestibulaire de la couronne de 33 et confirme sa situation symphysaire basse. L'examen tomodensitométrique (fig. 4) permet de déterminer la position exacte de 33, ses rapports avec les racines des incisives mandibulaires et sa position par rapport aux corticales osseuses. Sur les coupes axiales et les reconstructions orthogonales (fig. $\mathbf{4}$ a et $\mathbf{4}$ b) l'élargissement du sac péricoronaire est confirmé. La dent est à distance des apex des incisives et on note en regard de la couronne une soufflure de la corticale vestibulaire. La reconstruction tomodensitométrique 3 dimensions (fig. 4 c) objective la position dans les 3 plans de l'espace de la canine et la perforation de la table externe ainsi que son extériorisation. La position en classe 2 de Mupparapu contre-indique le redressement orthodontique. De plus, face à la suspicion d'une transformation kystique du sac péricoronaire, l'indication d'extraction est posée.

Tableau I

Classification de Mupparapu 2002 [3]

Classification de Mupparapu (2002)

CLASSE 1 La canine a une position oblique dans la région symphysaire. Sa pointe cuspidienne franchit la ligne médiane

CLASSE 2 En position horizontale, la dent est incluse à proximité du bord basilaire

CLASSE 3 La couronne de la dent incluse se situe au niveau de la canine controlatérale

CLASSE 4 position horizontale, la canine est placé
des molaires, du côté opposé de l'arcade

CLASSE 5 La canine 


\section{Situation clinique 1}
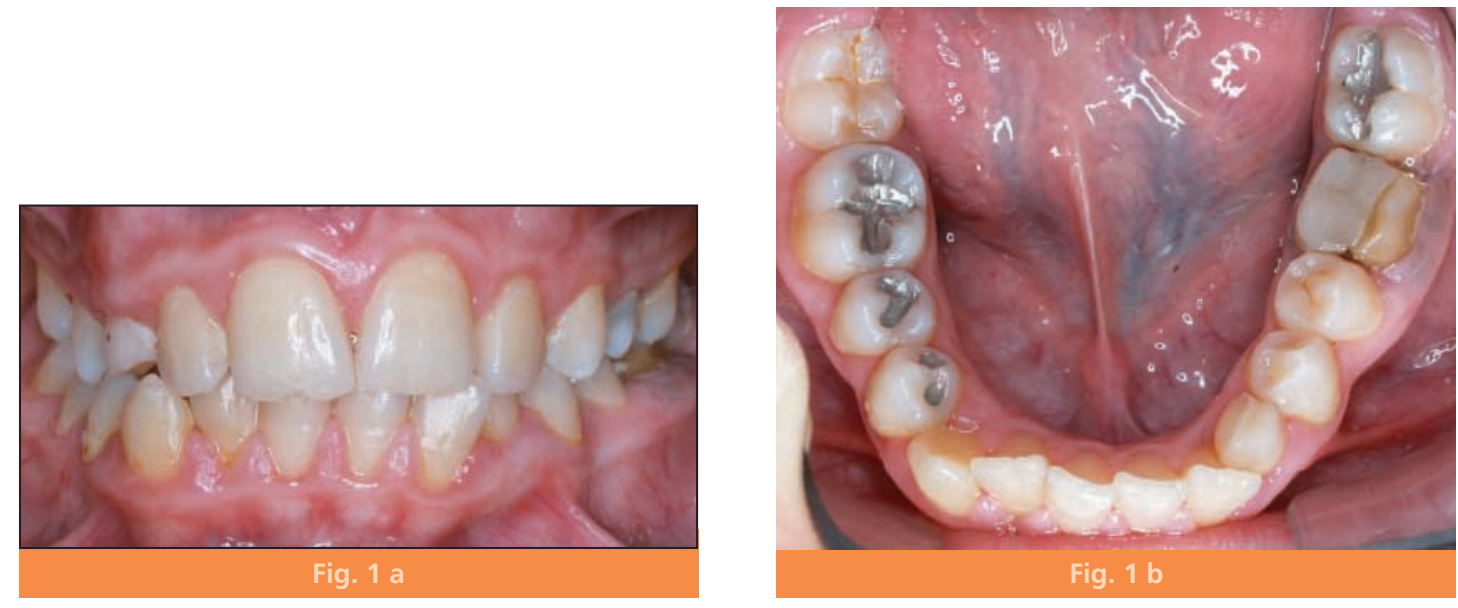

Fig. 1 a et 1 b Examen clinique endobuccal.

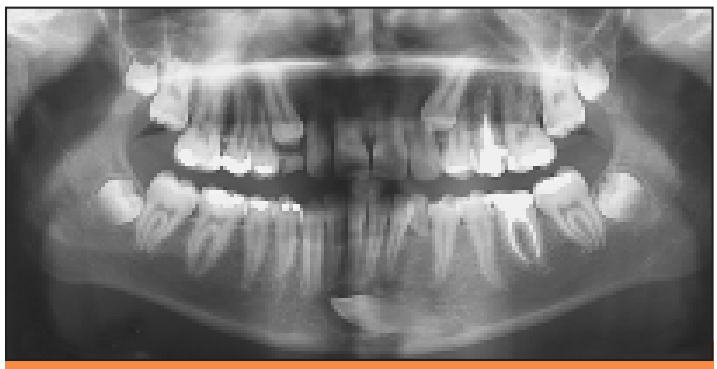

Fig. 2 Radiographie panoramique.

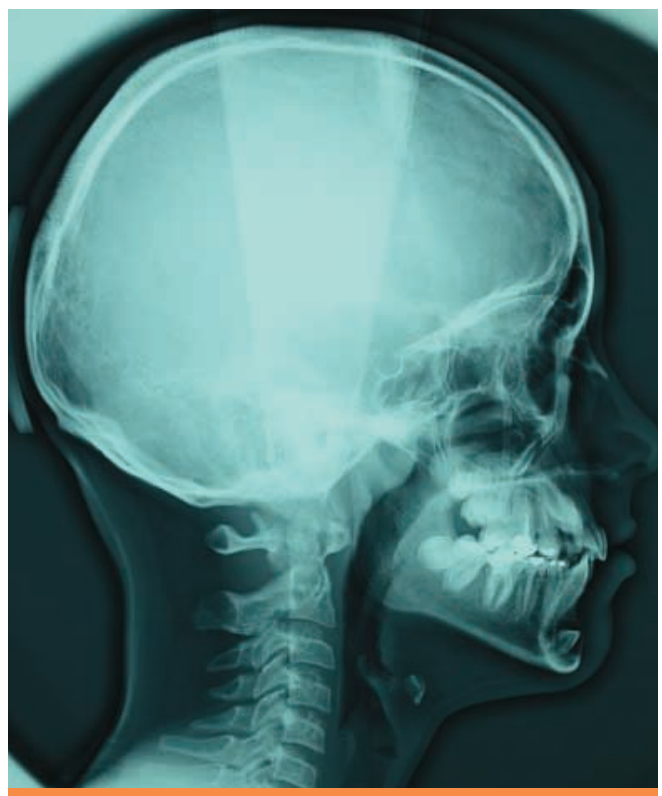

Fig. 3 Téléradiographie de profil. 


\section{Situation clinique 1}
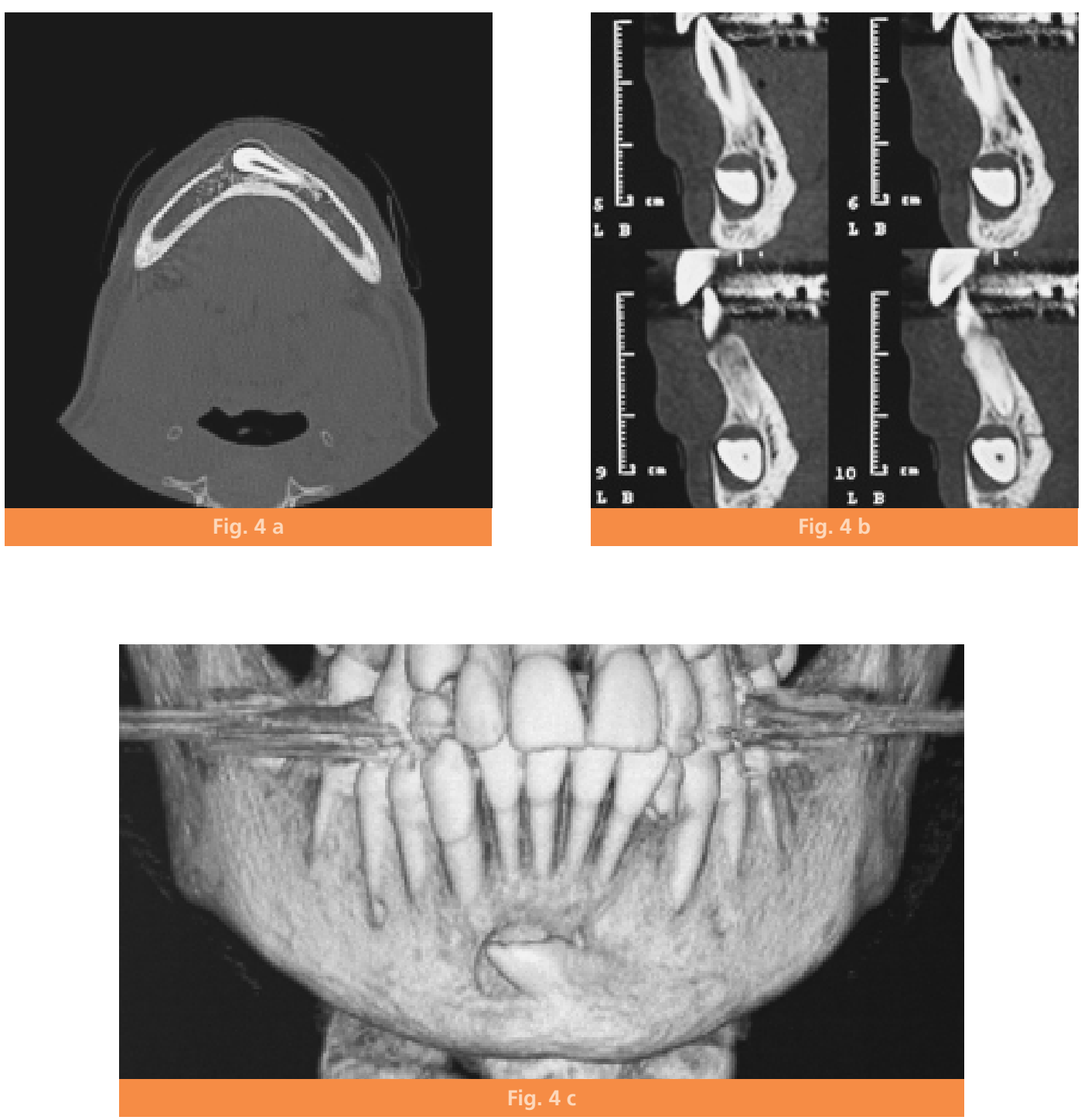

Fig. 4 a à c Examen tomodensitométrique

a : coupe axiale ;

b : reconstructions orthogonales ;

c: reconstruction tomodensitométrique 3 dimensions. 


\section{Situation clinique 1}

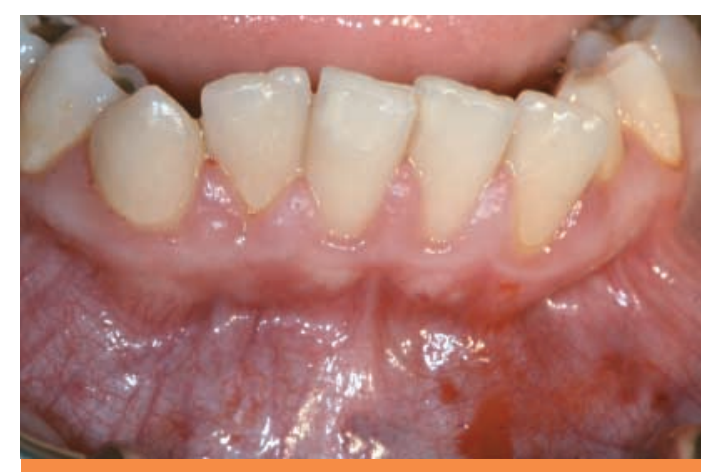

Fig. 5 a Situation initiale.

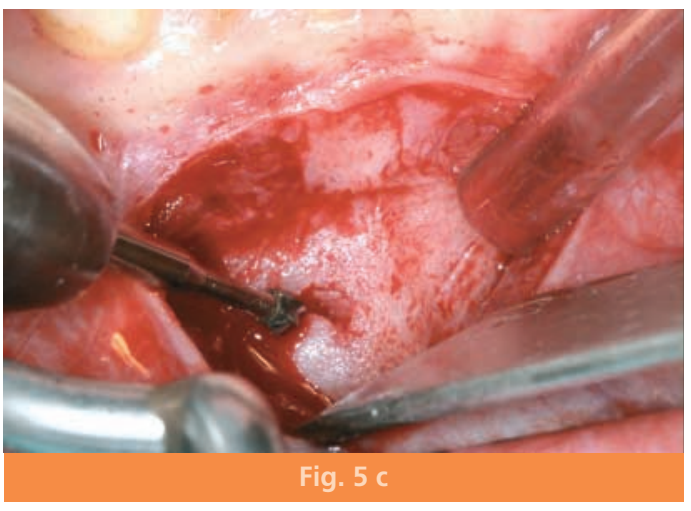

Fig. 5 c et d Temps osseux.
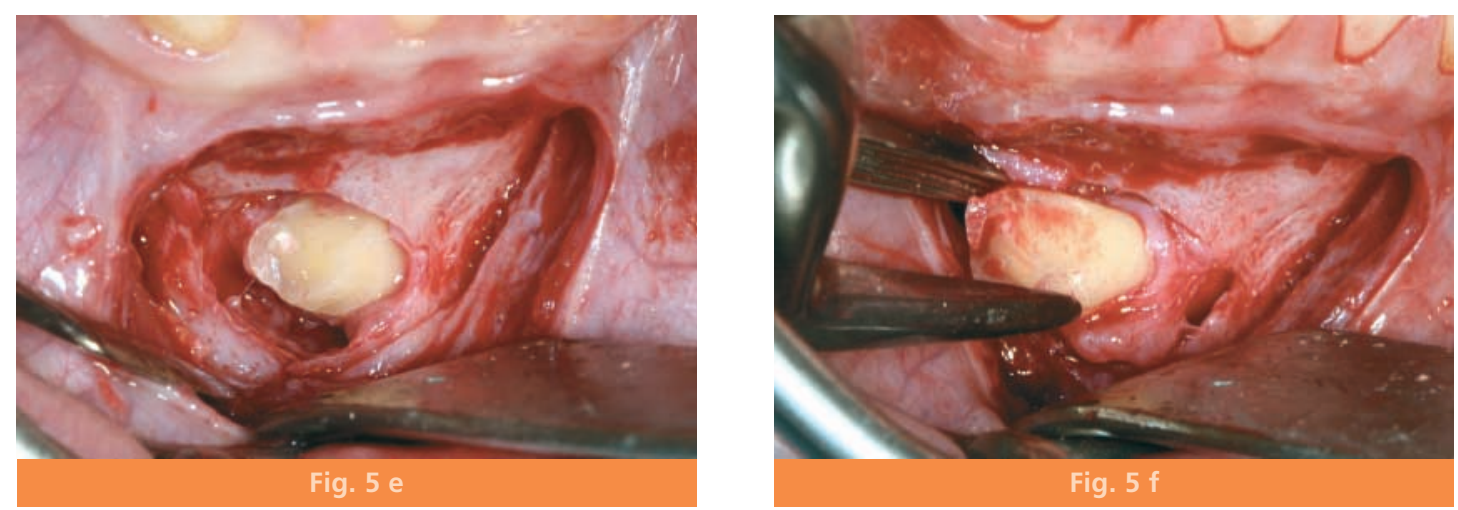

Fig. 5 e et $f$ Temps dentaire.

Fig. 5 a à $f$ Intervention chirurgicale.
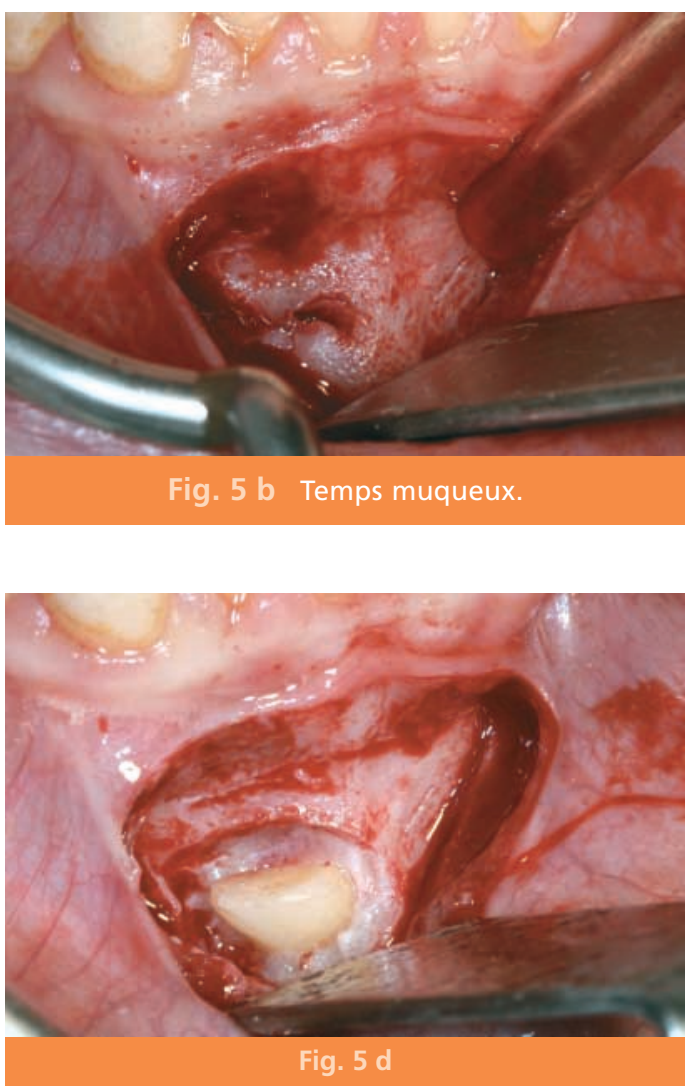


\section{Situation clinique 1}
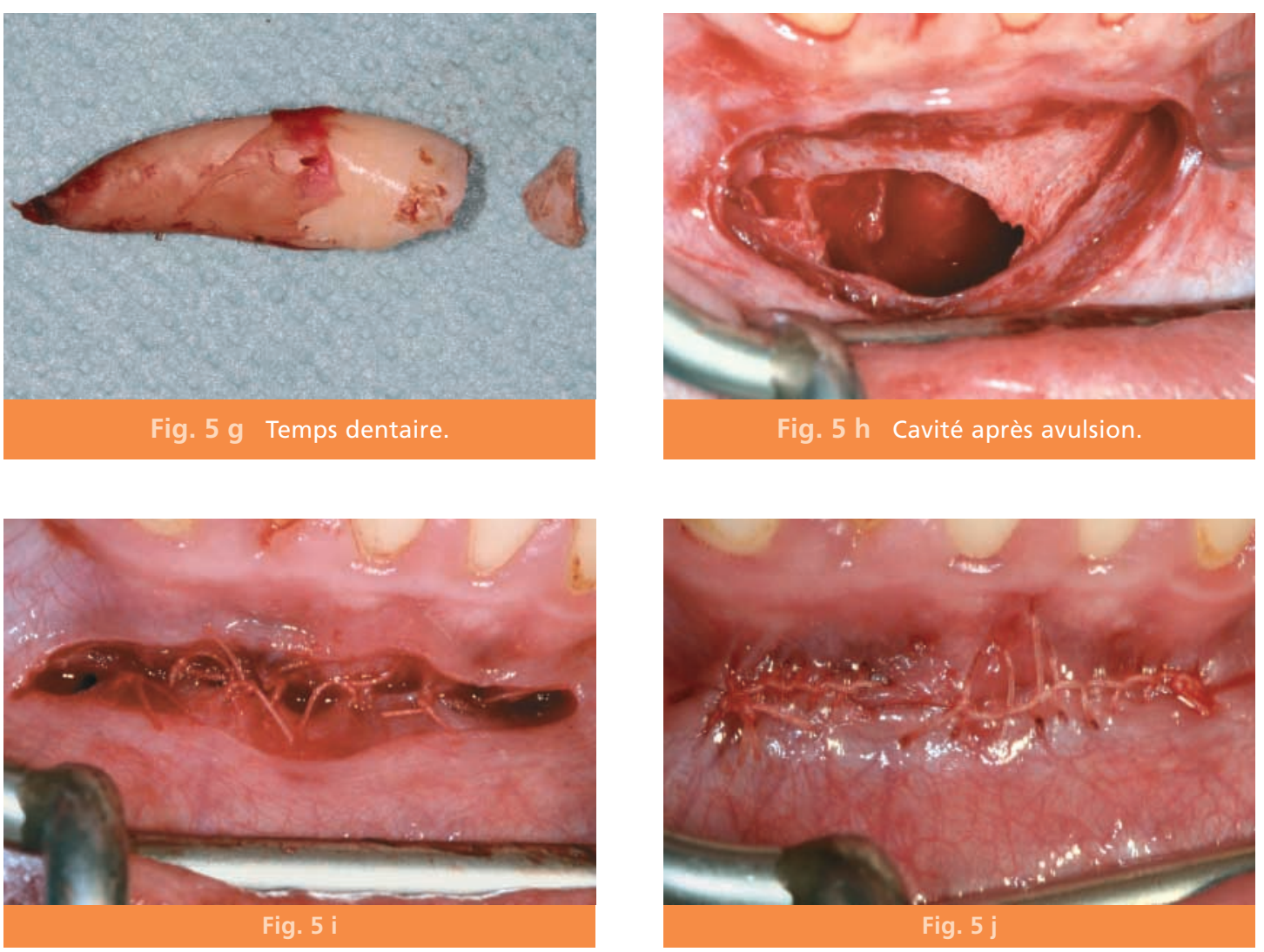

Fig. 5 i et j Sutures en deux plans.

Fig. 5 a à $f$ Intervention chirurgicale.

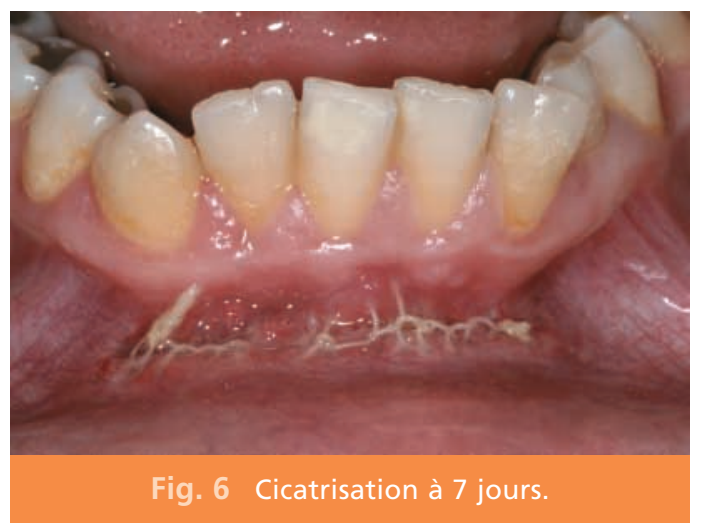


$>$ Intervention chirurgicale (fig. 5 a à j)

\section{- Anesthésie :}

L'anesthésie loco-régionale aux foramens mentonniers est pratiquée avec des rappels linguaux au niveau des incisives mandibulaires.

\section{- Temps muqueux :}

Une incision de canine à canine dans le vestibule à distance des collets est réalisée. La fibromuqueuse est décollée prudemment et un lambeau d'épaisseur totale est écarté permettant l'accès à la voussure de la corticale vestibulaire en regard des apex de 41 et 42 (fig. 5 b).

\section{- Temps osseux :}

Une ostéotomie à l'aide d'une fraise boule chirurgicale montée sur pièce à main au niveau de la voussure permet de dégager la couronne de 33 (fig. 5 c, 5 d). Le sac péricoronaire est retiré à l'aide de curettes et d'une pince d'Halstead-Mosquito.

\section{- Temps dentaire :}

La pointe de la canine est sectionnée avec une fraise zekria chirurgicale montée sur turbine (fig. 5 e). La dent est mobilisée à l'aide d'un syndesmotome faucille et avulsée avec un davier à racine maxillaire (fig. 5 f, 5 g).

\section{- Temps alvéolaire :}

La cavité est soigneusement nettoyée et les bords sont régularisés avec une râpe à os (fig. 5 h).

\section{- Sutures :}

Le lambeau est suturé sur deux plans. Le plan profond est d'abord suturé (fig. 5 i) avec des points séparés à l'aide d'un fil résorbable (Vicryl rapide 5.0). Puis le plan superficiel est suturé (fig. $\mathbf{5}$ j) par un surjet avec un fil résorbable de plus gros diamètre (Vicryl rapide 4.0 ).
Un traitement antalgique à base de paracétamol est prescrit ainsi qu'un bain de bouche antiseptique.

Le patient est contrôlé à une semaine, les suites opératoires ont été simples et la cicatrisation favorable (fig. 6).

\section{Situation clinique 2}

\section{> Présentation}

Un patient de 16 ans en cours de traitement orthodontique est adressé par son praticien pour avulsion de 43 incluse. L'examen clinique révèle l'absence de 43 sur l'arcade, une malposition de 33 et une hyperplasie gingivale du secteur antérieur mandibulaire (fig. 7). L'examen tomodensitométrique révèle une transmigration de classe 2 selon Mupparapu et une inclusion profonde de 43 à distance des apex des incisives. Les coupes axiales montrent un grand axe parallèle au rebord basilaire et une absence de déformation de la corticale. De plus, le sac péricoronaire est normal. Les coupes de reconstruction tomodensitométrique panoramique et orthogonales permettent de visualiser la profondeur de l'inclusion, le respect des apex dentaires et des corticales osseuses (fig. 8).

La position en classe 2 de 43 contre-indique la traction orthodontique et l'indication d'extraction est posée pour éviter une migration plus importante de la dent qui pourrait être risquée pour les apex dentaires.

\section{> Intervention chirurgicale (fig. 9 a à g)}

\section{- Anesthésie :}

L'intervention est pratiquée sous anesthésie générale. Des infiltrations locales sont réalisées pour faciliter le décollement, pour diminuer le saignement per-opératoire et la douleur post-opératoire. 


\section{Situation clinique 2}

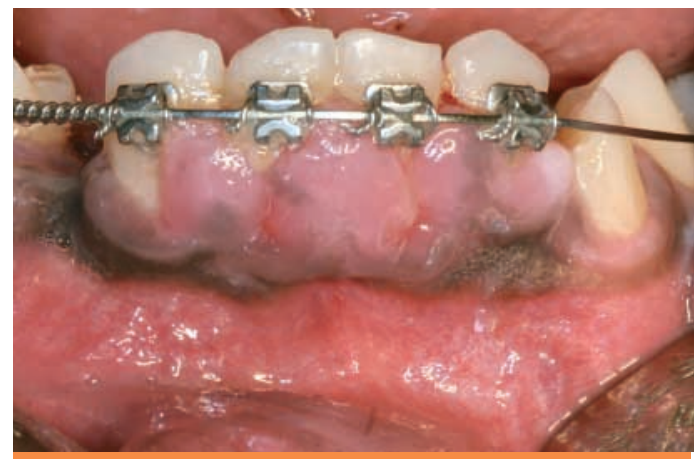

Fig. 7 Situation clinique 2, vue endobuccale.
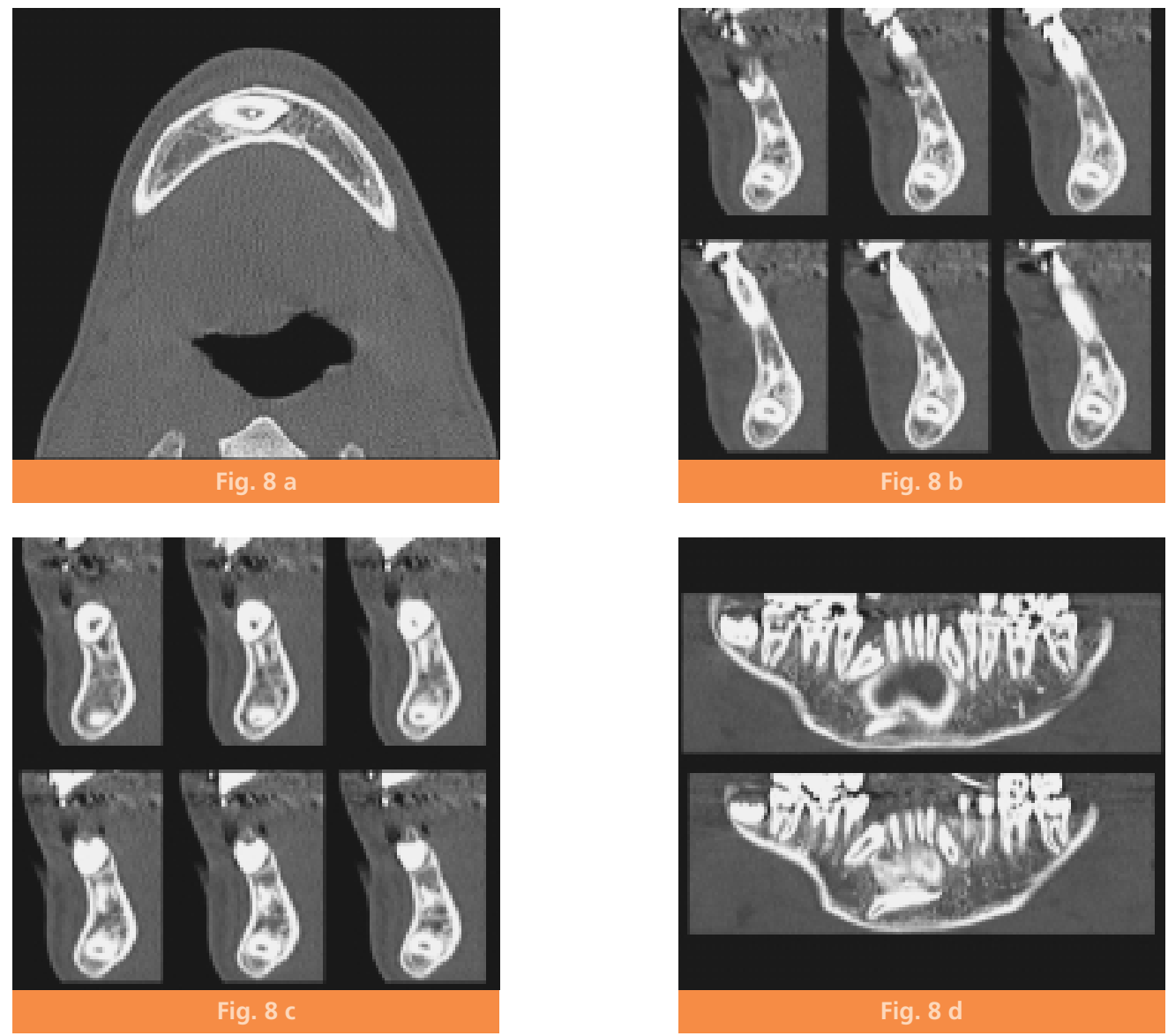

Fig. 8 a à $\mathrm{d}$ Examen tomodensitométrique

a : coupe axiale

b et c : reconstructions orthogonales ;

d : reconstruction tomodensitométrique panoramique. 
Situation clinique 2
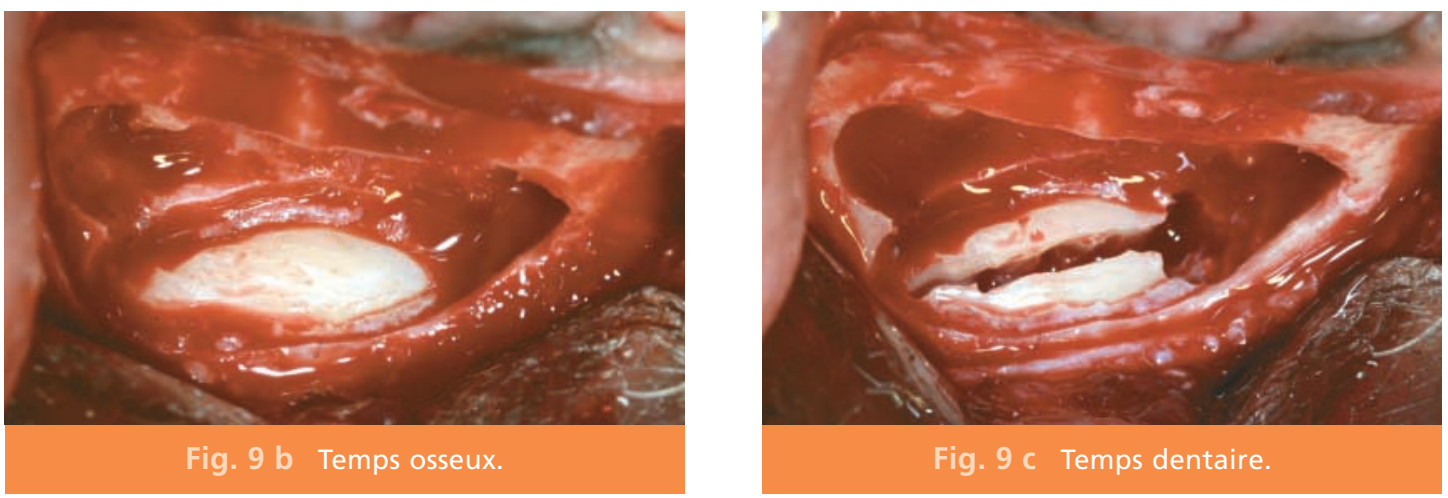

Fig. 9 c Temps dentaire.

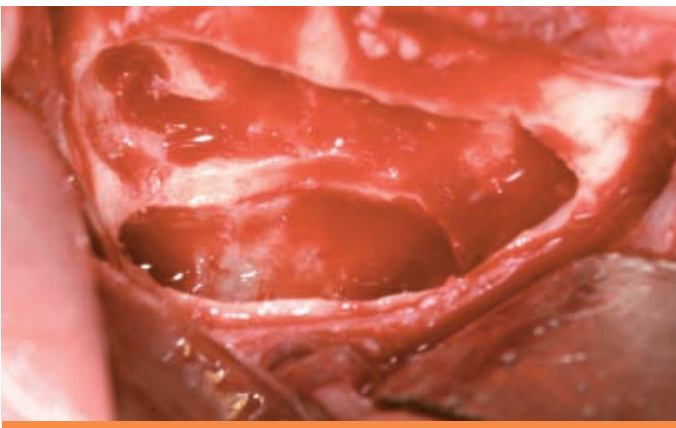

Fig. 9 d Cavité après extraction.
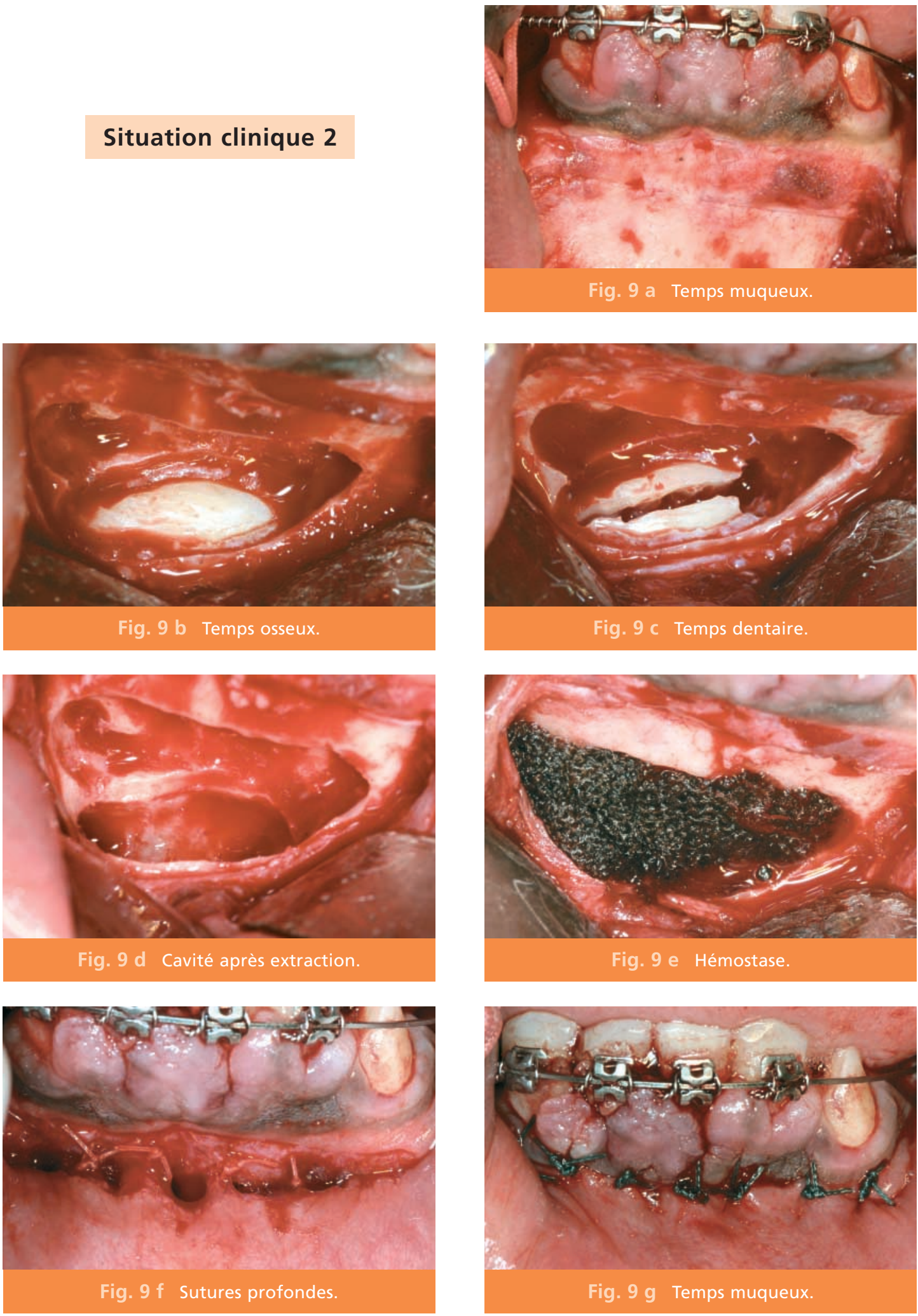

Fig. 9 a Temps muqueux.
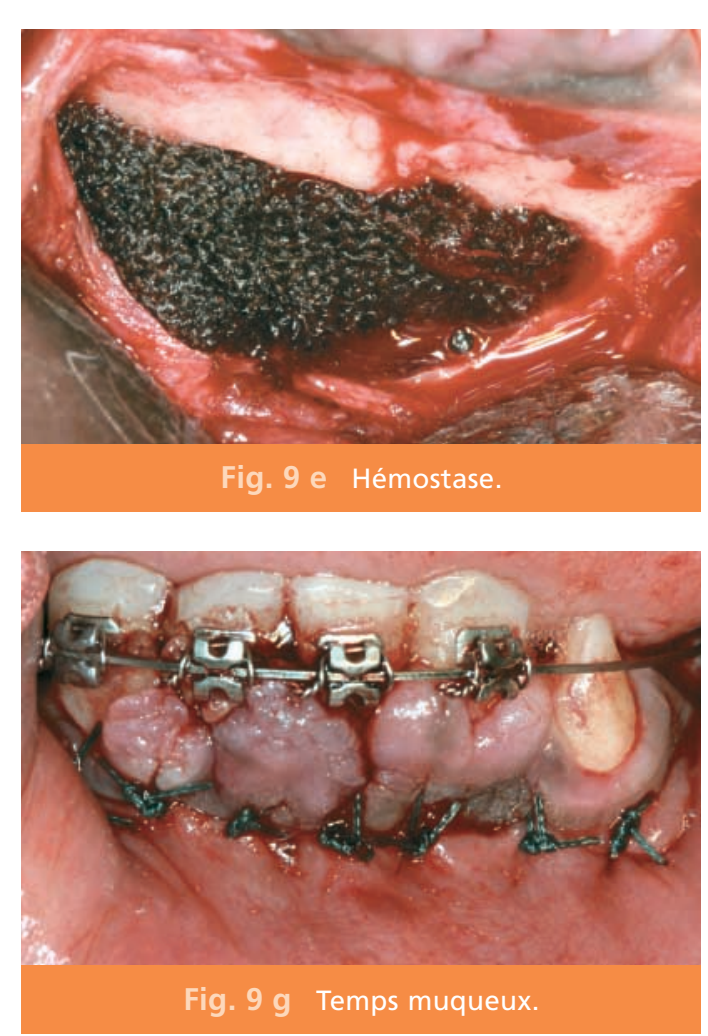

Fig. 9 a à $\mathrm{g}$ Intervention chirurgicale. 


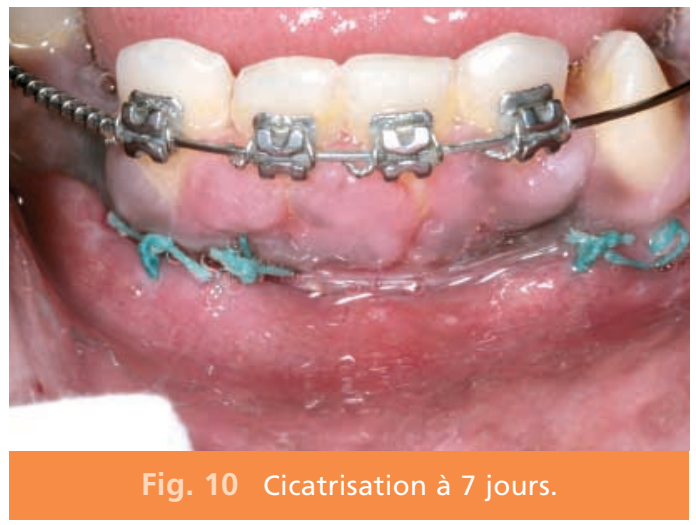

\section{- Temps muqueux :}

Une première incision en épaisseur partielle de canine à canine à distance des collets est pratiquée. Le lambeau est décollé délicatement en épaisseur partielle sur quelques millimètres puis une deuxième incision en épaisseur totale est réalisée. Il permet d'accéder à la zone symphysaire (fig. 9 a).

\section{- Temps osseux :}

Une fenêtre osseuse basse symphysaire est réalisée à la fraise boule montée sur pièce à main permettant de dégager la canine incluse horizontale (fig. 9 b).

\section{- Temps dentaire :}

La dent est sectionnée en trois parties à l'aide d'une fraise zékria montée sur pièce à main. Les trois fragments sont retirés (fig. 9 c, 9 d).

\section{- Temps alvéolaire :}

La cavité osseuse est soigneusement nettoyée, les bords sont régularisés. Une mèche d'oxycellulose (Surgicel ${ }^{\circledR}$ ) permet d'obtenir l'hémostase locale (fig. 9 e).

\section{Sutures:}

La suture est réalisée en deux plans, l'un musculaire profond (fig. 9 f) et l'autre muqueux superficiel (fig. 9 g).

\section{Situation clinique 2}

Un traitement antibiotique (amoxicilline $2 \mathrm{~g} . \mathrm{j}$-1 pendant 6 jours) associé à un traitement antalgique à base de paracétamol codéiné et à un bain de bouche antiseptique sont prescrits.

Le patient est contrôlé à une semaine, les suites opératoires ont été simples et la cicatrisation favorable (fig. 10).

\section{Discussion}

Le choix de l'anesthésie est fonction de plusieurs paramètres : la pusillanimité du patient, la profondeur d'inclusion, les difficultés opératoires et la longueur de l'intervention. Dans la situation clinique 1, la canine incluse était proche de la table osseuse externe, l'anatomie radiculaire ne présentait pas d'anomalies et le patient était coopérant ce qui nous a fait choisir l'anesthésie locale. Par contre, dans la situation 2, la canine était incluse profondément impliquant un fraisage osseux profond et une intervention longue, qui nous a fait opter pour l'anesthésie générale.

Le lambeau se situe dans les deux cas à distance des collets, dans le fond du vestibule, respectant ainsi le parodonte superficiel et profond du bloc incisif antérieur et permettant un dégagement large de la zone symphysaire. 
Le dégagement osseux doit être le moins délabrant possible et la section de la dent permet de limiter sa taille. Cependant, l'accès à la canine incluse profonde de la situation 2 a nécessité un délabrement important.

La zone symphysaire est richement vascularisée, ce qui peut entrainer des difficultés pour obtenir l'hémostase locale. Dans la situation 2, malgré des infiltrations locales avec vasoconstricteurs, le saignement per-opératoire a été important et l'hémostase locale a été difficile à obtenir nécessitant I'utilisation de mèches $d^{\prime}$ oxycellulose (Surgicel ${ }^{\circledR}$ ) en intra-cavitaire.

La zone mentonnière est riche en insertions musculaires et les sutures sont soumises à des tractions importantes. La suture en deux plans diminue le risque de déchirement et favorise une cicatrisation rapide. La suture profonde est pratiquée entre la face interne du lambeau et le périoste. La deuxième suture, superficielle, permet le rapprochement berge à berge des muqueuses. Dans la deuxième situation, deux incisions décalées ont été réalisées afin de faciliter la suture.

La prescription d'une antibiothérapie n'est pas systématique mais fonction du délabrement osseux et de la durée de l'intervention. Dans la situation 2, l'extraction de la dent incluse a nécessité un large accès osseux et une durée d'intervention importante ce qui nous a incité à prescrire une antibiothérapie.

\section{Extraction des canines incluses maxillaires}

La canine maxillaire est, après la dent de sagesse maxillaire, la dent la plus fréquemment incluse. L'incidence de l'inclusion des canines maxillaires est évaluée entre 1 et $3 \%$ de la population [4]. L'inclusion est unilatérale dans la majorité des cas. Dans le plan transversal, elle est plus souvent palatine (50\%) que vestibulaire $(30 \%)$ et présente une position intermédiaire dans $20 \%$ des cas. Dans le plan vertical, la canine est plus fréquemment située entre l'apex et le collet de l'incisive latérale ; seulement $10 \%$ des canines incluses ont une position plus apicale que les apex des incisives latérales.

De nombreuses causes de l'inclusion des canines maxillaires sont évoquées. Le caractère héréditaire est parfois avancé ainsi que les fac- teurs anatomiques qui conditionnent la migration de la dent durant son parcours intraosseux [2]. L'encombrement dentaire et le manque de place sur l'arcade n'expliquent qu'en partie l'inclusion canine. En effet, environ $85 \%$ des canines incluses maxillaires palatines présentent suffisamment d'espace pour leur éruption, tandis que seulement $17 \%$ des canines incluses en position vestibulaire ont un espace suffisant pour leur éruption [5]. Le redressement progressif des axes des incisives se produit pendant l'éruption de la canine. La morphologie et le stade de formation de l'incisive latérale servant de guide pour la canine peuvent être à l'origine d'une déviation palatine. Au contraire, un encombrement antérieur associant une réduction de l'espace inter- 
canin et une inclinaison distale de la couronne des incisives latérales va favoriser une évolution vestibulaire [2]. En outre, les canines vestibulaires ont une angulation plutôt verticale tandis que les palatines sont plus horizontales [5].

L'extraction de la canine incluse maxillaire est indiquée en cas de rotation de $180^{\circ}$ du germe, en cas d'ankylose aboutissant à l'échec du traitement orthodontique, en cas de processus infectieux ou tumoral volumineux et en cas de refus du traitement orthodontique.

\section{Situation clinique 3}

\section{$>$ Présentation}

Il s'agit d'une patiente de 35 ans en bonne santé, qui présente des épisodes infectieux palatins récurrents en regard de 12 . À la palpation palatine, on remarque la présence d'une voussure douloureuse (fig. 11). La radiographie panoramique objective la présence de 13 incluse, semblant résorber l'apex de 12 . Sa couronne est entourée d'un halo radioclair correspondant probablement à une formation kystique péricoronaire (fig. 12 a). Nous prescrivons un cliché occlusal qui semble nous indiquer la position palatine de 13 (fig. 12 b). L'examen tomodensitométrique confirme la position palatine de la canine et permet de préciser la profondeur d'inclusion. Sur les coupes axiales et les reconstructions orthogonales (fig. 13 a, 13 b), nous notons une rupture de la corticale palatine ainsi que l'absence de résorption radiculaire. La reconstruction en trois dimensions permet d'objectiver l'absence de contact direct de 13 avec les dents adjacentes (fig. 13 c). L'absence de motivation pour un traitement orthodontique ainsi que les récidives infectieuses fréquentes font poser l'indication d'extraction de 13 .

\section{> Intervention chirurgicale}

\section{- Anesthésie :}

L'anesthésie de la muqueuse palatine est réalisée par infiltration en regard de 16 et au niveau de foramen incisif. Elle est complétée par une anesthésie intra-cavitaire de 13 et par des rappels vestibulaires de 14 à 23 .

\section{- Temps muqueux :}

Une incision intrasulculaire palatine de 14 à 23 est réalisée. La fibromuqueuse est décollée prudemment et un lambeau d'épaisseur totale est chargé permettant l'accès à la voussure de la corticale palatine en regard des apex de 12 et 14, provoquée par la couronne de 13. On distingue alors une petite surface corticale perforée par la pointe de la canine (fig. 14 a,14 b).

\section{- Temps osseux :}

Une ostéotomie à l'aide d'une fraise boule chirurgicale montée sur pièce à main au niveau de la voussure permet de dégager la couronne de 13. Le sac péricoronaire et le tissu inflammatoire associé sont retirés à l'aide d'une pince gouge (fig. $14 \mathrm{c}$ ).

\section{- Temps dentaire :}

Deux tranchées sont réalisées avec une fraise zekria chirurgicale montée sur turbine dans la couronne de la canine. Puis la couronne est fragmentée en deux parties à l'aide d'un syndesmotome droit glissé dans les tranchées. La couronne de la canine est sectionnée en deux parties. Les deux morceaux coronaires sont alors extraits avec une pince de Halstead dont la courbure et la finesse de ses mords permettent une prise aisée. Le reste de la dent est mobilisé à l'aide d'un syndesmotome faucille et extrait avec un davier racine maxillaire (fig. $14 \mathrm{~d}, 14 \mathrm{e}$ ). 


\section{Situation clinique 3}

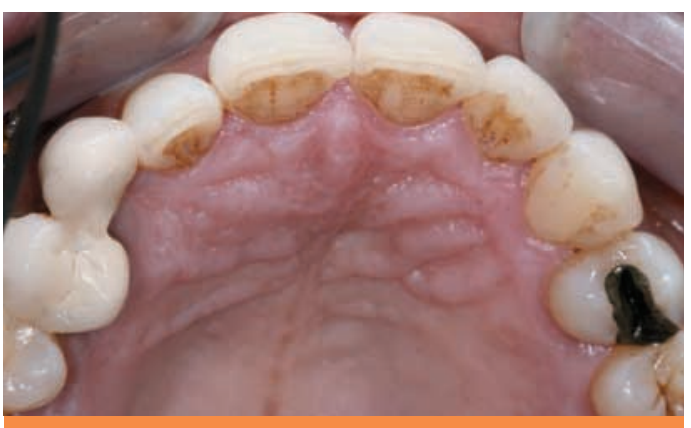

Fig. 11 Situation clinique initiale, vue endobuccale.

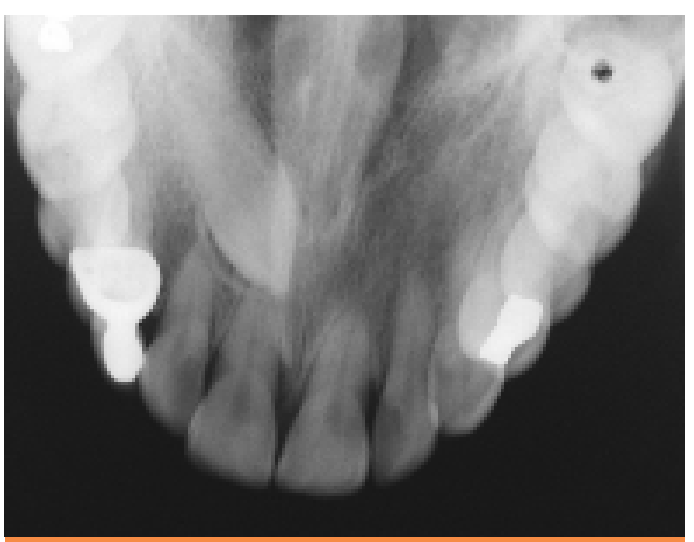

Fig. 12 b Cliché occlusal.

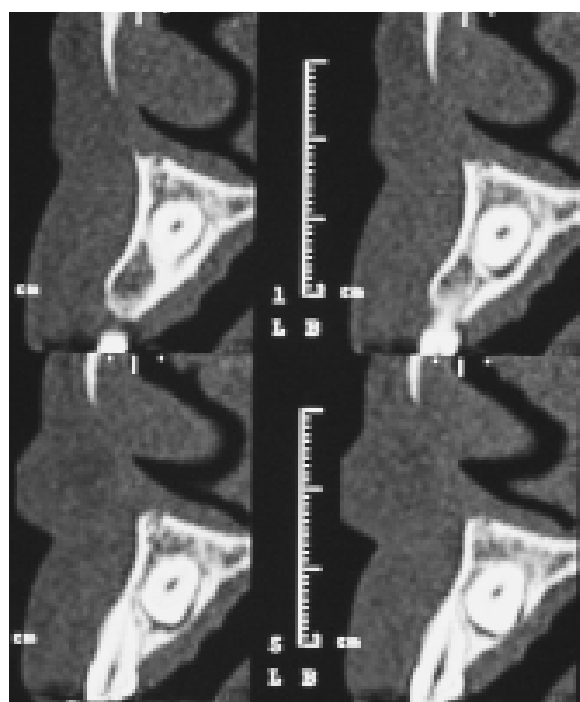

Fig. 13 b Reconstructions orthogonales.

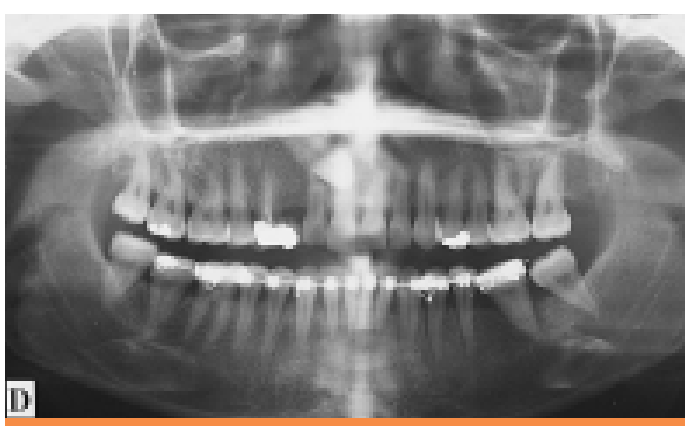

Fig. 12 a Radiographie panoramique.

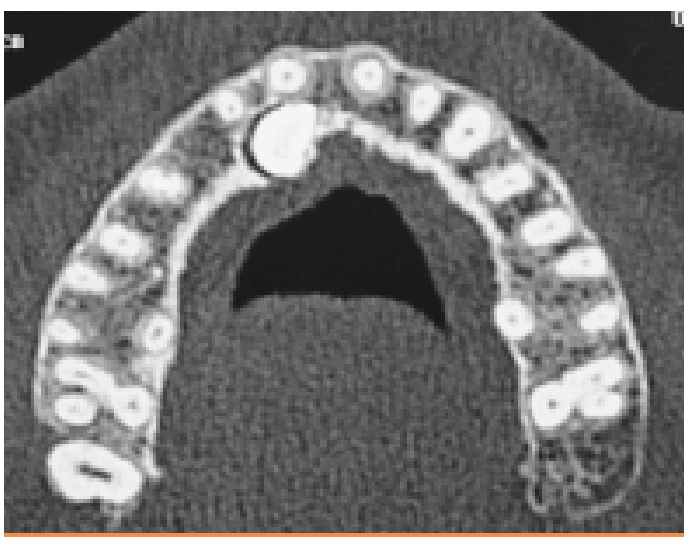

Fig. 13 a Examen tomodensitométrique coupe axiale.

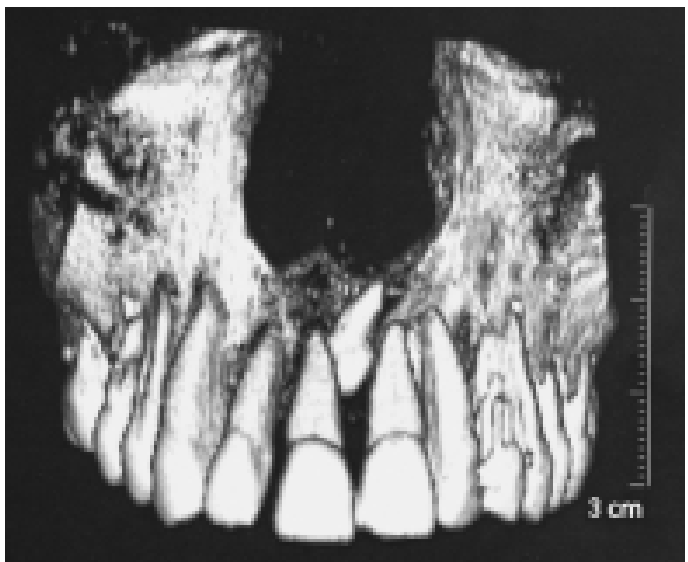

Fig. 13 c Reconstruction en trois dimensions. 


\section{Situation clinique 3}

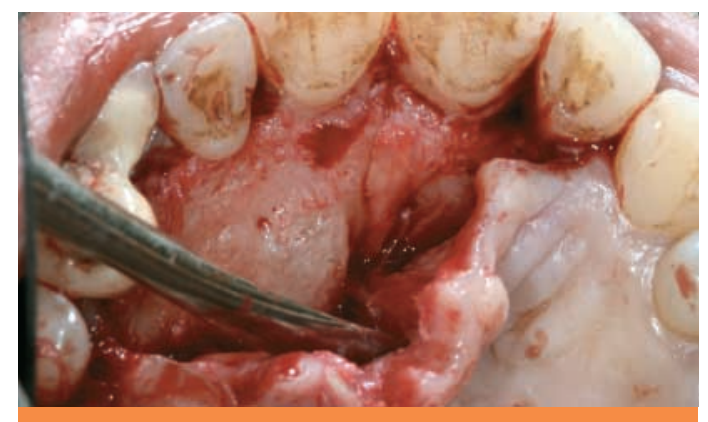

Fig. 14 a Temps muqueux.

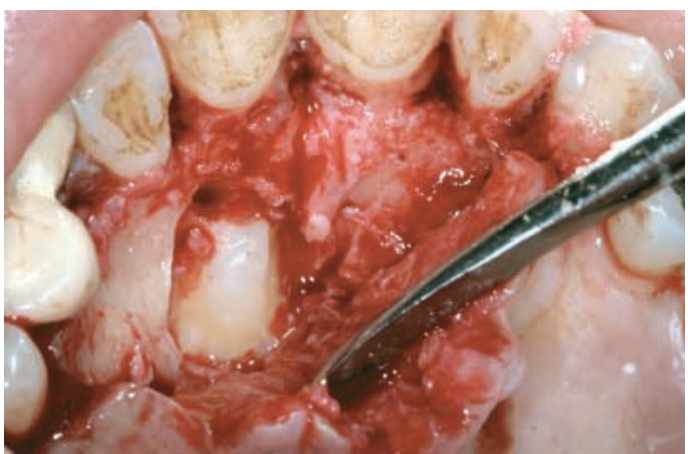

Fig. 14 c Temps osseux.

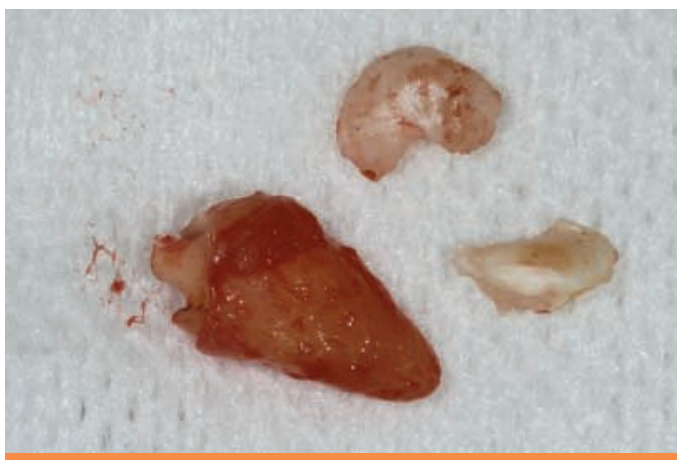

Fig. 14 e Temps dentaire.

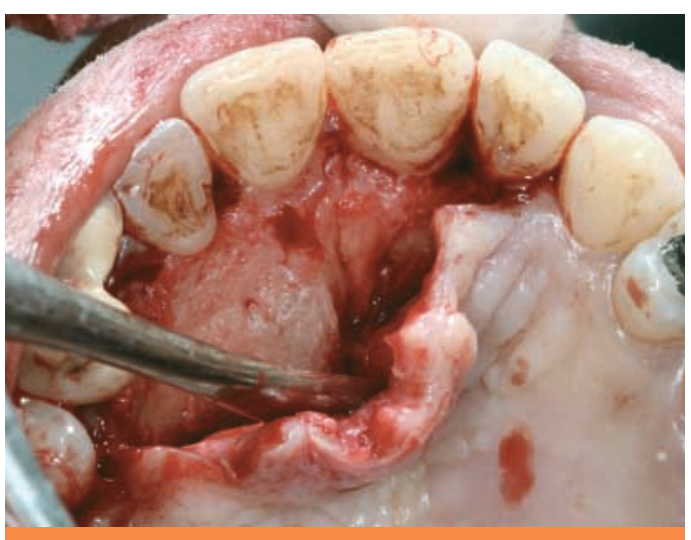

Fig. 14 b Temps muqueux.

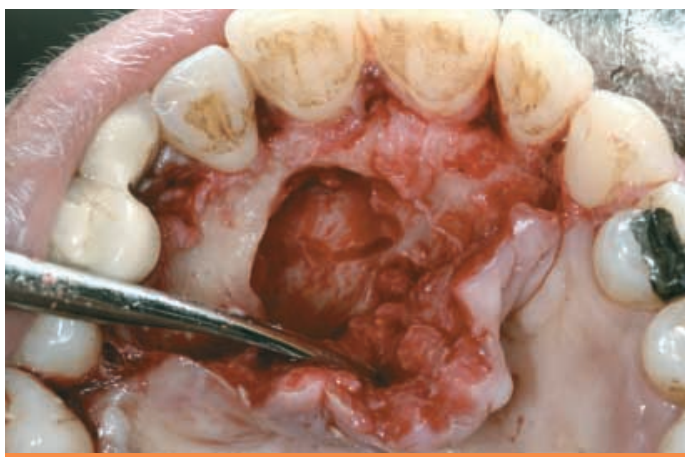

Fig. 14 d Temps dentaire.

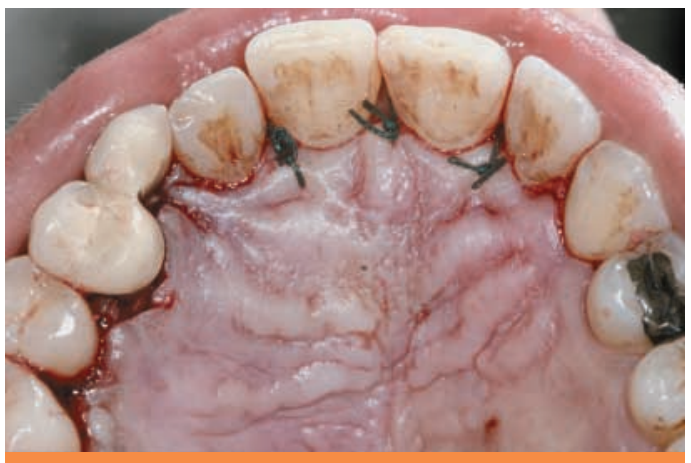

Fig. $14 \mathrm{f}$ Sutures.

Fig. 14 a à $f$ Intervention chirurgicale. 


\section{- Temps alvéolaire :}

La cavité est soigneusement nettoyée et les bords sont régularisés à l'aide de la fraise boule à os utilisée à vitesse lente.

\section{- Sutures :}

Le lambeau est suturé par des points inter-dentaires avec du fil Ethibond 3.0 non résorbable (fig. 14 f). L'hémostase obtenue, nous donnons des conseils post-opératoires à la patiente et instaurons un traitement antibiotique (amoxi- cilline $2 \mathrm{~g} / \mathrm{j}$ pendant 6 jours) et un traitement antalgique à base de paracétamol. La patiente est invitée à faire des bains de bouche antiseptiques à partir du lendemain de l'intervention. Elle est revue en contrôle à une semaine, la cicatrisation est favorable.

Ultérieurement, nous prévoyons la dépose du bridge, la réfection de la couronne sur 14 après reprise de traitement endodontique ainsi que la réalisation d'un implant en place de 13 après cicatrisation osseuse.

\section{Conclusion}

La conservation des canines incluses et leur mise en place sur l'arcade par des techniques de chirurgie et d'orthodontie n'est pas toujours possible. Les techniques d'extraction des ces dents visent à préserver le maximum de volume osseux afin de pouvoir poser éventuel- lement un implant dans les meilleurs conditions. La proximité étroite fréquente de ces canines avec les racines des incisives ou prémolaires et les structures anatomiques voisines peuvent amener à la prescription d'un examen tomodensitométrique.

\section{Bibliographie}

1. Aydin U, Yilmaz HH, Yildirim D.

Incidence

of canine impaction and transmigration in a patient population. Dentomaxillofac Radiol 2004 May;33(3):164-16.

2. Korbendau JM, Patti A. Réussir le traitement orthodontique et chirurgical des dents incluses.
Quintessence International. 2005.

3. Mupparapu M. Patterns of intra-osseous transmigration and ectopic eruption of mandibular canines: Review of literature and report of nine additional cases. Dentomaxillofac Radiol 2002 Nov;31(6):355-360. Review.
4. Bishara SE. Clinical management of impacted maxillary canines. Semin Orthod 1998 Jun;4(2):87-98. Review.

5. Cooke J, Wang HL. Canine impactions: incidence and management. Int J Periodontics Restorative Dent 2006 Oct;26(5):483-91. 


\section{SUMMARY}

\section{Extraction of impacted canine}

Marc BARANES

Marie LAVAUD

Louis MAMAN

\section{Keywords}

- impacted canine

- dental ectopy

- mental symphysis
The impaction of canines is a situation usually observed. The management is multidisciplinary. It requires proficiency in surgery and orthodontics. When extraction is indicated, a clinical and radiographic analysis must be made for check the exact position of this canine and relationship with teeth and structures nearby. The choice of anaesthesia technique and surgical proceeding will be make functions of analysis of these parameters.

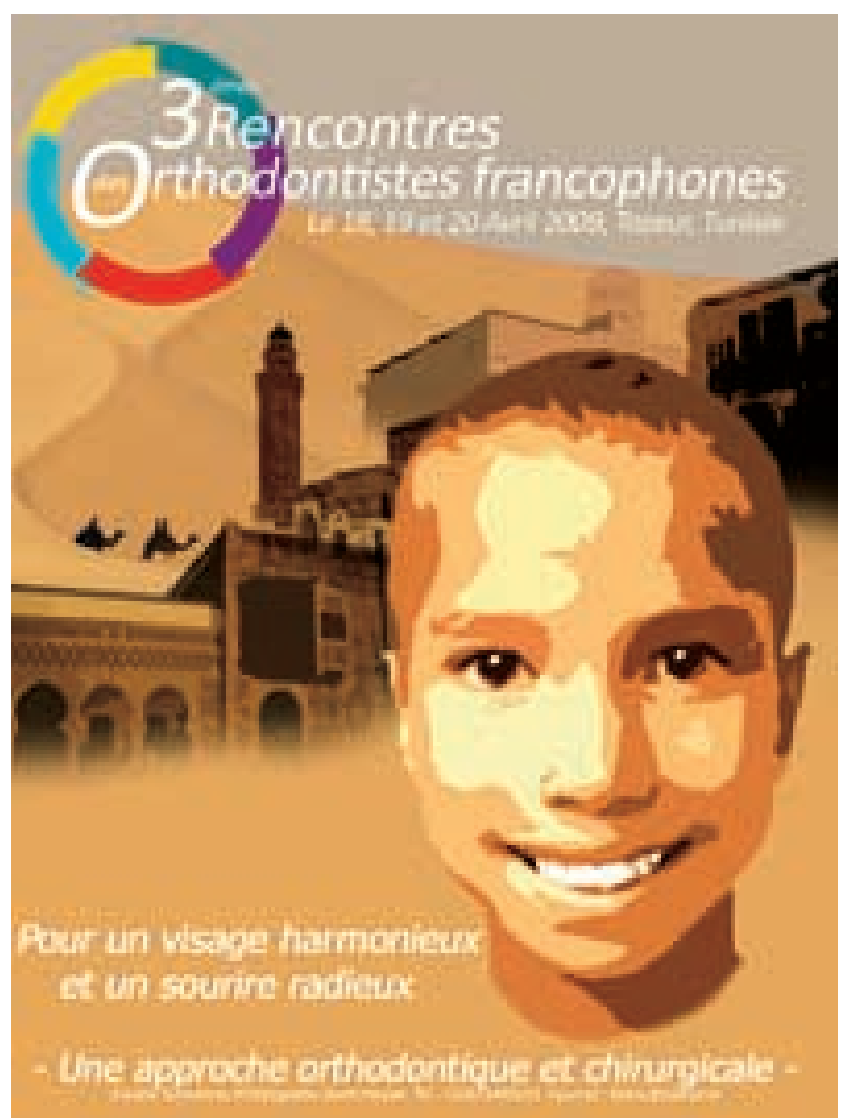




\section{LA VIE DE L'ASSOCIATION}

PRÉSIDENTS D'HONNEUR :

Pierre CERNÉA ${ }^{\dagger}$, Jean OUVRARD ${ }^{\dagger}$, Michel BENOIST ${ }^{\dagger}$

MEMBRES D'HONNEUR :

A. RICHARD ${ }^{\dagger}$, H. LENTULO ${ }^{\dagger}$,

A. LAMBERT, P. GONON ${ }^{\dagger}$,

J.-P. RAGOT ${ }^{\dagger}$

Ch. DESCROZAILLES,

G. FICHELLE

\section{CONSEIL D'ADMINISTRATION}

PRÉSIDENT : Hubert OUVRARD

\section{VICE-PRÉSIDENTS :}

LUC CHIKHANI,

Jean-François LEGRAND

SECRÉTAIRE GÉNÉRAL :

Frédéric LARCHÉ

TRÉSORIER :

Gérard PASQUET

\section{MEMBRES :}

Jacques-Charles BERTRAND,

Danielle BUCH, Jean BUQUET,

Estelle FAVRE, Jean MEYER,

Guy PRINC, Valérie TRAVERT

\begin{abstract}
COMITÉ SCIENTIFIQUE
PRÉSIDENT : LUC CHIKHANI

VICE-PRÉSIDENT : Hubert OUVRARD

SECRÉTAIRE : Frédéric LARCHÉ

MEMBRES : Christophe BONNEFOY, Estelle FAVRE, Gilles FLEURIDAS, Thierry GUÉRIN, Jean-François LEGRAND, Frank LEVAVASSEUR, Hervé MISSISTRANO, Gérard PASQUET, Thierry PIRAL, Valérie TRAVERT
\end{abstract}

ANCIENS PRÉSIDENTS : P. CERNÉA ${ }^{\dagger}$, P. HENNION, H. LENTULO ${ }^{\dagger}$, M. LEPOIVRE ${ }^{\dagger}$, A. MARMASSE ${ }^{\dagger}$, A. LAMBERT, R.-R. RIGOLET ${ }^{\dagger}$, R. BATAILLE ${ }^{\dagger}$, L.-A. STIEGLER ${ }^{\dagger}$, F. BOUCHON ${ }^{\dagger}$, Ch. DESCROZAILLES, P. FRIEZ ${ }^{\dagger}$, F. BROCHERE , A. RICHARD ${ }^{\dagger}$, R.-L. NINET ${ }^{\dagger}$, M. CHATEAU, P. COUSTAING, A. MUGNIER, R. WEILL ${ }^{\dagger}$, C. CREPY, J. OUVRARD ${ }^{\dagger}$, Y. COMMISSIONAT, B. DANGY ${ }^{\dagger}$, F. GARLOPEAU ${ }^{\dagger}$, J.-L. DEPHILIPPE, J. VIGNEUL, H. PETIT, M. BENOIST', G. FICHELLE, J. LAUFER, J.-P. SANTORO, P. LAUDENBACH ${ }^{\dagger}$, P. DARGENT ${ }^{\dagger}$, CI. SCHUHMANN, F. MAESTRONI ${ }^{\dagger}$, J.-P. DEFFEZ, H. OUVRARD, D. RIGOLET, J. BUQUET, J.$\begin{array}{ll}\text { P. RAGOT } & \\ & \text { R. BUGUGNANI, P. BORDAIS, }\end{array}$ J.-M. LAURICHESSE' ${ }^{\dagger}$, J.-Ch. BERTRAND, J.-F. LEGRAND, G. PASQUET, J. DICHAMP, D. BUCH, A. DEBOISE' C. BOZON, F. LARCHÉ, M. AMORIC, G. PRINC, V. TRAVERT, J.-P. LÉZY, F. LEVAVASSEUR, E. FAVRE, H. MISSISTRANO, T. PIRAL, C. BONNEFOY

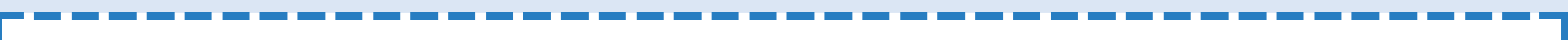
BULLETIN D'ADHÉSION À L'A.E.O.S.

Oui, je souhaite devenir membre de l'A.E.O.S. Ci-joint le règlement de ma cotisation annuelle 2008: $20 €$ au lieu de $60 €$ (offre réservée aux nouveaux abonnés des A.O.S.).

Je règle par chèque à l'ordre de I'A.E.O.S.

Dr:

Prénom :

Adresse

Code postal :

Ville :

Téléphone :

Fax :

Bulletin à retourner à : I'Association d'Enseignement d'Odontologie et de Stomatologie (A.E.O.S.) 179, rue Saint-Honoré - 75001 Paris - Tél. 0142605032 - Fax 0147034643 